ARTICLE

\title{
Cation-controlled wetting properties of vermiculite membranes and its promise for fouling resistant oil-water separation
}

\author{
K. Huang ${ }^{1,2}$, P. Rowe ${ }^{3}$, C. Chi ${ }^{1,2}$, V. Sreepal ${ }^{1,2}$, T. Bohn ${ }^{1,2}$, K.-G. Zhou ${ }^{1,2,4}$, Y. Su ${ }^{1,2,5}$, E. Prestat ${ }^{6}$,

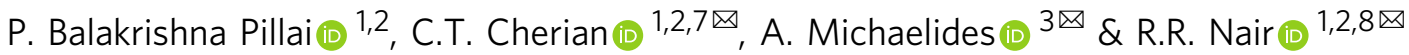

Manipulating the surface energy, and thereby the wetting properties of solids, has promise for various physical, chemical, biological and industrial processes. Typically, this is achieved by either chemical modification or by controlling the hierarchical structures of surfaces. Here we report a phenomenon whereby the wetting properties of vermiculite laminates are controlled by the hydrated cations on the surface and in the interlamellar space. We find that vermiculite laminates can be tuned from superhydrophilic to hydrophobic simply by exchanging the cations; hydrophilicity decreases with increasing cation hydration free energy, except for lithium. The lithium-exchanged vermiculite laminate is found to provide a superhydrophilic surface due to its anomalous hydrated structure at the vermiculite surface. Building on these findings, we demonstrate the potential application of superhydrophilic lithium exchanged vermiculite as a thin coating layer on microfiltration membranes to resist fouling, and thus, we address a major challenge for oil-water separation technology.

\footnotetext{
${ }^{1}$ National Graphene Institute, University of Manchester, Manchester M13 9PL, UK. ${ }^{2}$ Department of Chemical Engineering and Analytical Science, University of Manchester, Manchester M13 9PL, UK. ${ }^{3}$ Thomas Young Centre, London Centre for Nanotechnology, and Department of Physics and Astronomy, University College London, Gower Street, London WC1E 6BT, UK. ${ }^{4}$ Institute of Molecular Plus, Tianjin University, Tianjin 300072, China. ${ }^{5}$ Department of Materials, Loughborough University, Loughborough LE11 3TU, UK. ${ }^{6}$ Department of Materials, University of Manchester, Manchester M13 9PL, UK.

7 Department of Physics and Electronics, CHRIST (Deemed to be University), Bangalore 560029, India. ${ }^{8}$ Henry Royce Institute for Advanced Materials,

Oxford Road, Manchester M13 9PL, UK.凶email: christie.thomas@christuniversity.in; angelos.michaelides@ucl.ac.uk; rahul@manchester.ac.uk
} 
H ydrated ions at charged interfaces are ubiquitous and play a critical role in our daily life ranging from the control of neurological action, protein folding, to clean energy and water production ${ }^{1-4}$. Clays are one of the best examples of such systems ${ }^{5}$. Owing to their unique properties such as high capillarity, degree of swelling 6 , and exceptional thermal and chemical stability, they are widely used in sectors ranging from cosmetics, medicine, agriculture, oil and gas industries, environmental remediation and construction engineering ${ }^{6-8}$. Among the many different types of synthetic and naturally occurring clays, vermiculite is a natural clay mineral that has a layered structure with two silica tetrahedral sheets fused to an edge shared octahedral sheet of magnesium, iron or aluminium hydroxides ${ }^{9}$. The stacking of the layers generates regular van der Waals gaps, which are filled with exchangeable hydrated cations ${ }^{10,11}$. Compared with other clays, vermiculite has higher cation exchange capacity, greater layer charge density, and limited swelling ${ }^{10,12}$, making it an ideal candidate for developing membranes. Even though it has been an extensively researched material for decades, the microscopic understanding of its wetting properties or its exploitation as a membrane is limited. Previous studies on the wetting properties of clays suggest that the wetting properties are linked to its surface charge, the nature of interlayer cations, interlayer water content, and the surface topography ${ }^{13-15}$. As far as the effect of exchangeable cations is concerned, a few studies on kaolinite, a non-swelling clay mineral, revealed conflicting results ranging from negligible impact to cation-dependent wettability $13,14,16$. The inconclusive nature of the previous wettability studies could be due to the particulate nature of the clays and the difficulty in obtaining a smooth surface for water contact angle measurements. Given the importance of surface charge on the wetting properties of solids ${ }^{17,18}$, it is plausible that the adsorption of ions to the surfaces or interfaces could affect the wetting properties of solids ${ }^{19}$. However, previous studies reveal that even though it can influence the wetting properties, a large wetting transition from hydrophilic to hydrophobic has not been observed.
The development of superwetting materials that can be used to modify the performance of membranes for ultra/microfiltration has recently gained increased attention because of the potential it holds to reduce membrane fouling, and so address a major challenge in e.g. the area of oil-water separation ${ }^{20-24}$. Most studies reported so far have focused on the surface modification of metallic mesh and textile membranes with superwetting materials. Due to the large pore size of these membranes, this can only be applied for the separation of immiscible oil/water mixtures at lower pressures (mainly under gravity) and is not suitable for emulsions ${ }^{21,22}$. There have also been attempts to modify the surfaces of ultra/microfiltration polymeric membranes by superwetting materials, but due to the instability of the hydration layer upon prolonged exposure to fouling environments, these membranes were found to be unsuitable for continuous filtration ${ }^{21,22}$.

In this paper, we successfully demonstrate the wetting transition of vermiculite laminates (V-laminates) from superhydrophilic to hydrophobic by cation exchange technique. We show that the wetting properties of vermiculite laminates are sensitive to the atomic scale details of how the ions are hydrated. The Li-exchanged vermiculite laminate is found to provide a stable superhydrophilic surface. Further, we also demonstrate the potential application of superhydrophilic lithium vermiculate (LiV) for fouling resistant oil-water separation by a simple and scalable coating of superhydrophilic LiV on polymeric microfiltration membranes. The superhydrophilic LiV-coated membrane exhibits significantly improved flux and fouling resistance compared with the noncoated or other superhydrophilic polymer-coated membranes when separating both immiscible oil and water mixture and emulsion.

\section{Results}

Characterisation of vermiculite laminates. Stable aqueous dispersions of $\mathrm{LiV}$ (inset of Fig. 1a) flakes were prepared from thermally expanded vermiculite crystals using a reflux ion exchange methodology as reported previously 9,11 (Methods). The transmission electron microscopy (TEM) (Fig. 1a) and atomic a

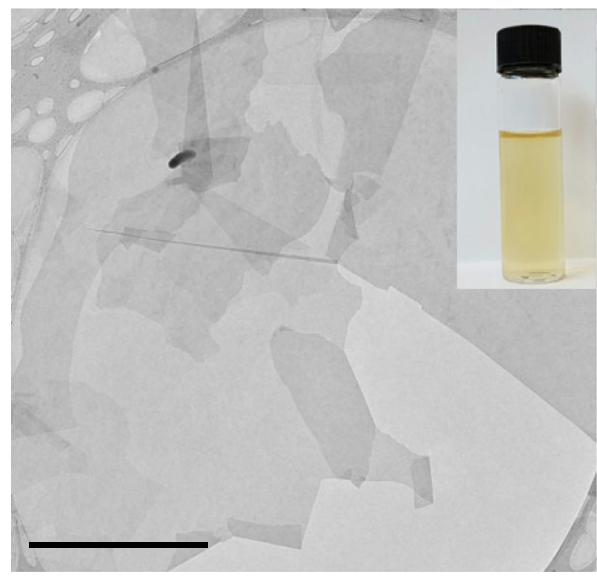

b

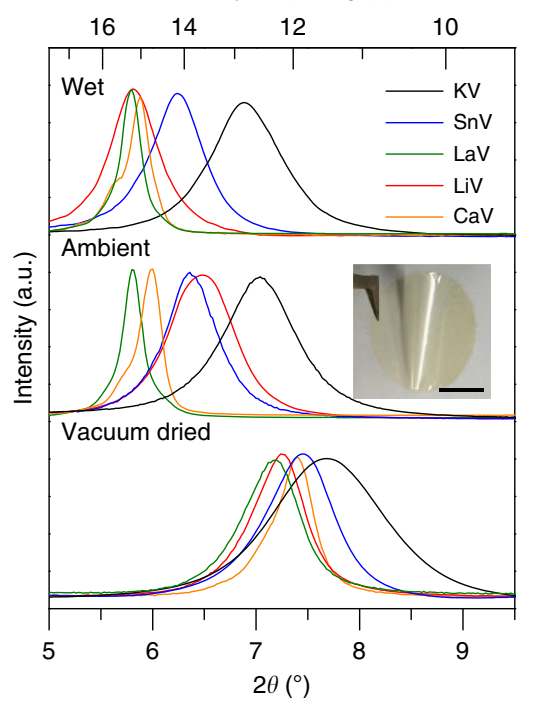

Fig. 1 Cation exchanged vermiculite laminates. a Transmission electron microscope (TEM) image of exfoliated lithium vermiculite flakes. Scale bar, 10 um. Inset: Photograph of an aqueous dispersion of exfoliated vermiculite. b X-ray diffraction (XRD) pattern of free-standing K-, Sn-, La-, Li-, and Ca-vermiculite laminates in vacuum dried ( $12 \mathrm{~h}$ ), ambient ( $\sim 40 \%$ relative humidity) and wet states. Source data are provided as a Source Data file. Inset: Photo of a 5 $\mu \mathrm{m}$-thick free-standing Li-vermiculite laminate. Scale bar, $1 \mathrm{~cm}$. It is noteworthy that the distribution of interlayer spacing of ion-exchanged vermiculite laminates significantly changes after its exposure to water or humid air. This suggests that the hydration of the membrane influences the quality of the laminar structure of the membrane. 
a

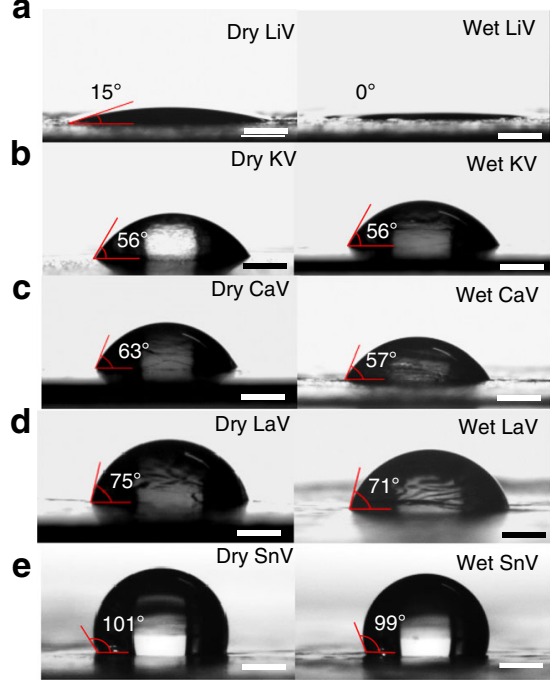

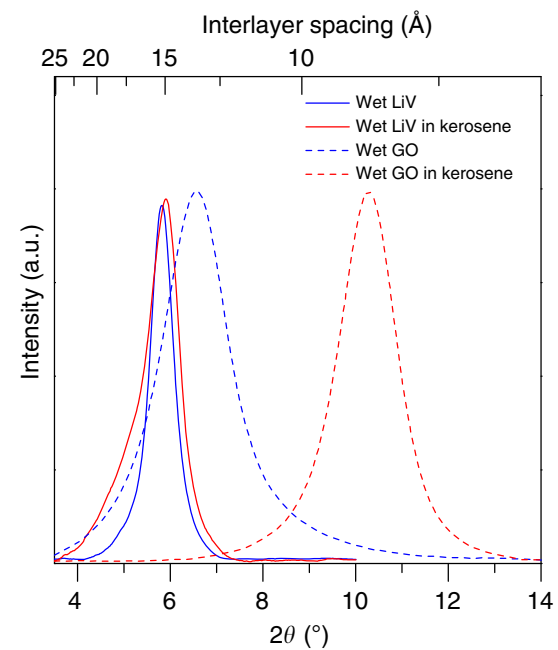

Fig. 2 Wetting properties of vermiculite laminates. a-e Water contact angle of lithium vermiculite (LiV), potassium vermiculite (KV), calcium vermiculite $(\mathrm{CaV})$, lanthanum vermiculite ( $\mathrm{LaV})$, and tin vermiculite $(\mathrm{SnV})$-laminates in dry and wet states. Scale bar, $750 \mu \mathrm{m}$. We also measured the water contact angle for sodium vermiculite $(\mathrm{NaV})$ and obtained a similar value $\left(52 \pm 1^{\circ}\right)$ to that of $\mathrm{KV}$ (Supplementary Fig. 5). $\mathbf{f}$ Evaluation of water pinning property of free-standing wet LiV laminate measured by capturing XRD from wet LiV laminate before and after immersing in kerosene for a week. As a reference, a hydrophilic graphene oxide (GO) laminate was also tested in the same experimental conditions, it was found that water molecules were released from the GO membrane after $12 \mathrm{~h}$ of kerosene exposure. Source data are provided as a Source Data file.

force microscopy (AFM) images (Supplementary Fig. 1) confirm that the individual flakes are defect free and have a thickness of $\approx 1.5 \mathrm{~nm}$. Micrometre-thick $(5 \mu \mathrm{m}) \mathrm{V}$-laminates (Fig. 1b) were prepared from the above dispersion using vacuum filtration as described in 'Methods'. We used a simple ion exchange method to replace $\mathrm{Li}$ ions in the interlayers of the vermiculite membranes to other desired cations such as potassium $\left(\mathrm{K}^{+}\right)$, calcium $\left(\mathrm{Ca}^{2+}\right)$, lanthanum $\left(\mathrm{La}^{3+}\right)$, and tin $\left(\mathrm{Sn}^{4+}\right)$ (Methods) ${ }^{10}$. Inductively coupled plasma mass spectrometry analysis confirms that Li has been completely interchanged with the desired cations (Supplementary Table 1).

It is well known that the cations in interlayer galleries of the bulk vermiculite crystals are hydrated and that the extent of hydration could depend on the competition of cation hydration energy and the electrostatic interaction between the cation and the layer charge ${ }^{25}$. Consistent with this, vermiculite laminates with different interlayer cations show different Interlayer spacing ( $d$-spacing) and different hydration behaviour. Figure $1 \mathrm{~b}$ shows $\mathrm{X}$-ray diffraction (XRD) of V-laminates vacuum dried for $12 \mathrm{~h}$, then exposed to air ( $\sim 40 \%$ relative humidity) and subsequently soaked in water (Methods). The measured $d$-spacings of vacuum dried samples are in the range of 12.2-11.5 $\AA$, typical of bulk vermiculite with a single hydration layer ${ }^{26}$. On the other hand, when exposed to ambient air the $d$-spacings increase to $12.6 \AA$, 13.6 $\AA$, $13.9 \AA, 14.7 \AA$ and $15.2 \AA$ for K-vermiculite $(\mathrm{KV}), \mathrm{Li}$ vermiculite $(\mathrm{LiV}), \mathrm{Sn}$-vermiculite $(\mathrm{SnV})$, Ca-vermiculite $(\mathrm{CaV})$, and La-vermiculite (LaV) laminates, respectively. Further exposure to liquid water only increases the $d$-spacing of $\mathrm{LiV}$ (13.6-15.2 $\mathrm{A}$ ), and no change is observed for $\mathrm{CaV}, \mathrm{LaV}, \mathrm{SnV}$ and $\mathrm{KV}$ laminates. The observed swelling behaviour of $\mathrm{V}$ laminates is consistent with swelling of bulk vermiculite ${ }^{26,27}$ and further confirms the exchange of $\mathrm{Li}$ cations to the other cations used.

Wetting properties of vermiculite laminates. Compared with the bulk vermiculite crystals one of the unique features of the exfoliated vermiculite is its potential for use as a membrane or coating. Hence a thorough understanding of the wetting properties of such films is essential. We have used contact angle measurements to characterise the surface wetting properties of $\mathrm{V}$ laminates. Figure $2 \mathrm{a}-\mathrm{e}$ show the water contact angle measurements on cation exchanged V-laminates in dry (ambient) and wet states. We find that cation-exchange modifies the wetting properties of $\mathrm{V}$-laminate in a tunable manner. The water contact angle in air for LiV-laminate is $15 \pm 1^{\circ}$ whereas it is $56 \pm 2^{\circ}, 63 \pm 3^{\circ}, 75$ $\pm 2^{\circ}$, and $101 \pm 2^{\circ}$ for $\mathrm{K}^{+}, \mathrm{Ca}^{2+}, \mathrm{La}^{3+}$, and $\mathrm{Sn}^{4+}$ exchanged laminates respectively. It is surprising to note that the $\mathrm{LiV}$ laminate is more hydrophilic compared with all other cation exchanged V-laminates and in the wet state, LiV-laminate is superhydrophilic with zero contact angle while all other $\mathrm{V}$ laminates retained the same wetting behaviour. To confirm that these effects are not due to the partial exchange of cations, we have also performed XRD and contact angle measurements as a function of salt concentration and salt solution exposure time during the ion exchange process. These measurements revealed that $60 \mathrm{~min}$ of $1 \mathrm{M}$ salt solution exposure is sufficient for the full ion exchange process (Supplementary Figs. 2 and 3). To further demonstrate the uniqueness of the observed cation controlled superhydrophilic to hydrophobic wetting transition in the $\mathrm{V}$ laminates, we have also performed similar experiments with graphene oxide (GO) membranes (Methods). Cation-modified GO membrane has been shown to offer excellent performance in terms of ion sieving ${ }^{28}$. However, in terms of the sensitivity of the wetting properties of GO to cation modification, we find only a small/moderate sensitivity of $\sim 15^{\circ}$ (Supplementary Fig. 4).

In general, superhydrophilic surfaces retain a hydration layer, and these layers function as a self-cleaning agent by repelling foulants such as biological molecules and oils $24,29,30$. One of the main drawbacks of such surfaces is the instability of the hydration layer upon prolonged exposure to fouling environments ${ }^{20,24,29}$ To investigate the hydration stability of LiV-laminates we have conducted XRD experiments on hydrated LiV-laminates exposed to oil. Figure $2 \mathrm{f}$ shows the stable hydration of LiV-laminate (no change in $d$-spacing) even after a week of kerosene soaking. To rule out the possibility of intercalation of oil molecules into the interlayers and hence providing the same $d$-spacing, we exposed a dry LiV-laminate to kerosene, where we did not observe any 
a

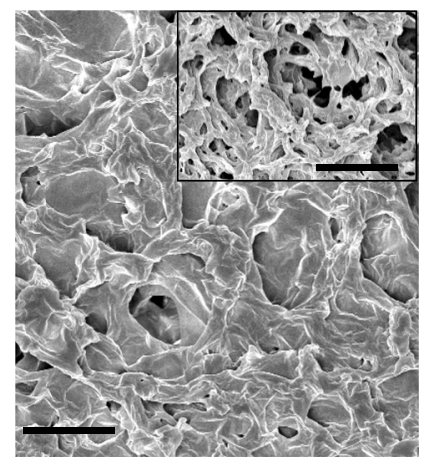

C

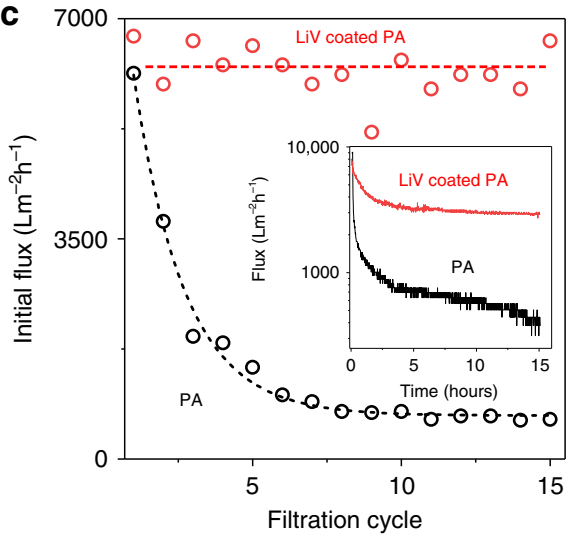

b
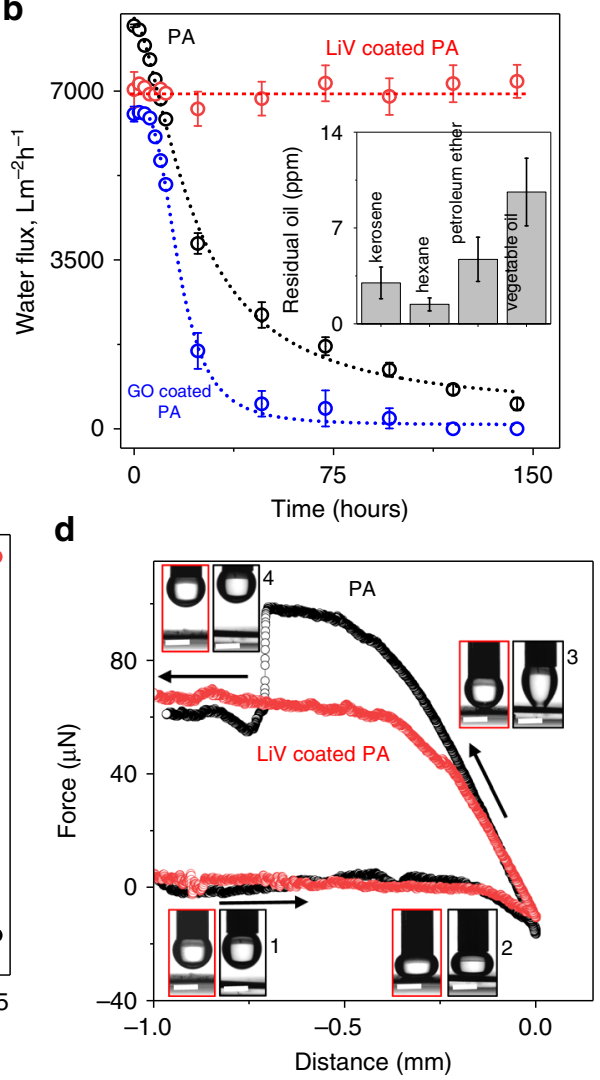

Fig. 3 Oil-water separation performance. a SEM image showing the topography of Li-vermiculite (LiV)-coated ( $\backsim 30 \mathrm{~nm})$ polyamide (PA) membrane. Scale bar is $1 \mu \mathrm{m}$. Inset: scanning electron microscopy (SEM) image of bare PA membrane. Scale bar; $3 \mu \mathrm{m}$. b Water flux through the LiV-coated PA, bare $\mathrm{PA}$, and a reference $\mathrm{GO}$-coated $(\backsim 30 \mathrm{~nm})$ PA membrane as a function of time during which membrane was in contact with kerosene. The measurement is performed at 1 bar differential pressure. Error bars denote standard deviations using three different measurements. Inset; residual organic carbon content in the permeate water of the LiV-coatedPA membrane for different types of oil. Error bars denote standard deviation using measurement at different time intervals. c Initial permeate flux at each filtration cycle during the multiple cycle emulsion separation by dead-end filtration at a pressure of 1 bar. The dotted lines are guides to the eye. Inset; Permeate flux through LiV-coated and bare PA during the cross-flow filtration of oil-in-water emulsion at 1 bar with a cross flow velocity of $0.05 \mathrm{~m} \mathrm{~s}^{-1}$. The initial decrease in permeate flux is due to the oil droplet deposition on to the membrane surface, and LiV coating significantly improves this flux decline and provides an approximately seven times higher steady-state flux compared with bare PA. d Force-distance curves recorded while the sample approaches and detaches from the oil droplet (colour coded labels). The adhesion force measurement process involves four major steps: (1) the sample surface approaches the oil droplet, (2) oil contacts the sample surface under a fixed preload, (3) sample surface leaves the oil droplet leading to deformation of the oil droplet due to oil-sample adhesion force, and (4) sample surface completely detaches from the oil droplet. Arrows indicate the direction of force measurement. Inset; Photographs showing the shape of the oil droplets during the force measurement for LiV-coated PA (red outline) and PA (black outline) at the corresponding stages. Scale bar $2 \mathrm{~mm}$. All source data are provided as a Source Data file.

intercalation (Supplementary Fig. 6). As a control experiment, we have also conducted similar tests with hydrated GO membranes, which have recently been suggested as an antifouling membrane for oil-water separation ${ }^{31}$. As shown in Fig.2f, the $d$-spacing of the wet GO membrane decreases from 13.5 to $8.5 \AA$, when exposed to kerosene for $12 \mathrm{~h}$. This suggests that the interlayer water molecules in the GO membrane have diffused into the oil and the GO membrane has become dry in the oil environment. This in turn implies that the interlayer water in the GO membranes is relatively mobile whereas in the LiV-laminates it is firmly pinned to the interlayer gallery.

Fouling resistant oil-water separation using $\mathrm{LiV}$ membrane. The exceptional water pinning features of LiV-laminate suggest its potential for designing antifouling oil-water separation membranes. To study the antifouling properties of $\mathrm{LiV}$, we deposited a thin layer of $\mathrm{LiV}$ on a hydrophilic microfiltration membrane (polyamide (PA) - nylon) with pore size $1.1 \mu \mathrm{m}$ (Fig. $3 \mathrm{a}$ and Methods) and performed oil-water filtration experiments. The LiV coating is non-continuous but homogeneous as shown by the SEM image (Supplementary Fig. 7). The pore size of such membranes was determined by capillary flow porometry (Methods). With increasing the LiV coating thickness, the pore size is observed to decrease and correspondingly the water flux through the membrane also decreases (Supplementary Fig. 8). For further antifouling studies, we optimised the coating thickness to $\sim 30 \mathrm{~nm}$ such that the wetting time is minimised and the water flux through the coated membrane is comparable to the bare PA membrane (>75\%) (Supplementary Fig. 8).

To test the antifouling properties of the membranes, we have then performed the oil-water separation experiments. Initially, all the membranes were pre-wetted with water to form the surface hydration layer to prevent the oil permeation ${ }^{29,30}$. Such water wetted membranes allow water to permeate but block oil entirely, up to the breakthrough pressure of the membrane ( 2 bar for the membranes reported in Fig. 3$)^{30}$. Water flux through such membranes which were continuously in contact with a column of oil $(50 \mathrm{~mL})$ was periodically monitored. Figure $3 \mathrm{~b}$ shows the water flux as a function of time for the LiV-coated PA membrane 
as well as the reference PA membrane and the GO-coated PA membrane. The LiV-coated membrane shows a remarkably stable water flux of $\sim 7000 \mathrm{~L} \mathrm{~m}^{-2} \mathrm{~h}^{-1}$ (at $1 \mathrm{bar}$ ) even after it is in continuous contact with oil for a week whereas the bare PA suffers severe fouling. The reference hydrophilic GO coating on $\mathrm{PA}$ is found to be resistant to fouling for an initial $6 \mathrm{~h}$ (Fig. 3b), but thereafter fouling happens even more severely than for the bare PA. We have also studied the oil repellent property of the $\mathrm{LiV}$-coated membrane by measuring the residual organic carbon content in the permeate water and found remarkably high oil repellency (few ppm carbon content as shown in Fig. 3b inset).

The observed antifouling properties of $\mathrm{LiV}$-coated membranes were found to be robust even for oil in water emulsion separations (Fig. $3 \mathrm{c}$ and Methods). Typically, superhydrophobic membranes are used for water in oil emulsion and superhydrophilic membranes are used for oil in water emulsion separation. For the later, oil droplets in the emulsion tend to adhere to the surface of the membrane and hence permanently foul the membranes after multiple filtration experiments ${ }^{21,22}$. Multiple cycles (15 cycles) dead-end filtration data in Fig. 3c shows that the initial water flux (Supplementary Fig. 9) of the LiV-coated membrane completely recovers after each cycle of filtration whereas for the reference PA membranes the water flux continuously deteriorated with increasing filtration cycle. This suggests that the adhesion of the oil droplets to the LiV coating is weak, and hence, a simple water rinsing is sufficient to lift the oil droplet from the surface of the membrane. This can be further corroborated from the stable water flux obtained for the LiVcoated PA membrane compared with a bare PA membrane in cross-flow filtration (Methods), where a tangential flow of the feed solution with even a smaller speed of $0.05 \mathrm{~m} \mathrm{~s}^{-1}$ detach the oil droplet from the surface and decreases the fouling. The steadystate permeate flux through LiV-coated PA membrane is found to be more than seven times higher than the bare PA and the flux increases with increasing cross-flow velocity (Supplementary Fig. 10). To quantify the adhesion force between the oil-droplet and the LiV coating we have measured the dynamic underwater oil-adhesion force (Fig. 3d) and oil droplet roll-off angles (Methods) and found that the adhesion force is below the detection limit $(<1 \mu \mathrm{N}$ for LiV-coated PA and $\sim 40 \mu \mathrm{N}$ for PA) and the roll-off angle is extremely small $\left(<3^{\circ}\right.$ for LiV-coated PA and $58.3^{\circ}$ for PA) (Supplementary Fig. 11).

To further demonstrate the significance and uniqueness of the LiV coating on the oil-water separation, we have compared the antifouling properties of the LiV coating with other common superhydrophilic coatings such as polydopamine and polyacrylamide hydrogel (see Methods). Even though both the polydopamine and hydrogel-coated PA showed an increased wettability from $1.4 \mathrm{~s}$ to $\sim 0.7 \mathrm{~s}$ similar to that of the vermiculite coating, they failed to provide long term antifouling properties (Supplementary Fig. 12). In addition, we have also measured the steady-state permeate flux and oil rejection of commercial microfiltration membranes polyethersulfone (PES), and polyvinylidene difluoride (PVDF) in cross-flow filtration before and after LiV coating. Compared with PA, both PES and PVDF were leaky to oil and LiV coating significantly enhances the oil rejection without hugely influencing the flux (Supplementary Figs. 13, 14) even for less hydrophilic PVDF membranes. In addition, we have investigated the effect of salt solution exposure on the antifouling properties of LiV-coated membranes and this shows that the addition of $\mathrm{NaCl}$ or $\mathrm{KCl}$ to the emulsion did not significantly affect the antifouling performance of the membranes for $100 \mathrm{~min}$ of continuous filtration (Supplementary Fig. 15). To probe this further, we have studied the wetting and oil droplet roll-off angle of LiV-coated PA membrane after the exposure of different salty emulsion solutions. We found that compared with bare PA, the wetting properties of mono- and divalent-cation exchanged membranes were similar, but the vermiculite coating significantly decreases the oil droplet roll-off angle and does not change significantly with mono- and divalent-cation exchange (Supplementary Fig. 16). This suggests that the weak interaction of oil droplets to the vermiculite surface also helps to improve the antifouling property of the membrane.

The robustness and adhesion between the LiV coating and the PA membrane have been investigated by measuring the change in wetting time and underwater oil contact angle of the LiV-coated PA membranes after the direct flush of water $\left(5 \mathrm{~L} \mathrm{~min}^{-1}\right.$, perpendicular to the membrane surface). The absence of any significant changes in wetting time and underwater oil contact angle with water flushing time (Supplementary Fig. 17) suggest that the adhesion of the LiV coating to the PA membrane is strong enough to withstand practical applications.

\section{Discussion}

The exceptionally stable antifouling properties of LiV-coated membranes could be attributed to the superhydrophilicity of LiV along with its water pinning properties. The superhydrophilic surface of LiV improves the water wetting and the underwater oleophobicity of PA (Supplementary Figs. 8, 18), whereas water pinning due to the hydration of $\mathrm{Li}$ ions in LiV maintains the membrane in a hydrated state thereby allowing it to retain its oilrepelling characteristics for a long time.

To test whether the unique superhydrophilicity of LiV is associated with surface charge density, we probed the ionic strength dependence of the contact angle (Methods) and found negligible change with ionic strength, suggesting that surface charge is not the crucial factor (Supplementary Fig. 19). To confirm this further, we have also measured the zeta potential of V-laminates, which is directly related to the surface charge density, and its dependence on the ionic strength. Our results show that the zeta-potential varies with ionic strength unlike that of the contact angle behaviour (Supplementary Fig. 19).

Further, we estimated the surface free energy from contact angle data using the primary equation of Fowkes' surface energy theory as detailed in 'Methods'. The dispersive component of surface energy is roughly the same for all vermiculite membranes irrespective of the intercalated cation whereas the polar component decreases in the order $\mathrm{LiV}>\mathrm{KV}>\mathrm{CaV}>\mathrm{LaV}>\mathrm{SnV}$ (Supplementary Fig. 20). Typically the polar component originates from Coulomb interactions between dipoles (e.g. hydrogen bond) ${ }^{32}$ and hence this suggests that the origin of the superhydrophilic properties of LiV could be linked to the hydrated structure of the $\mathrm{Li}$ cation.

To probe this further, we have performed density functional theory (DFT) calculations and ab initio molecular dynamics (AIMD) simulations (Methods). Here we choose to focus on thin water films on the surface of KV and LiV to clearly elucidate the cause of the anomalously hydrophilic nature of the LiV system.

In our analysis, we find that potassium ions exhibit a strong preference for adsorption on top of the hydroxyl groups present on the vermiculite basal plane. In the lithium case, there is a competition between two adsorption sites, one in which the lithium is chelated into the vermiculite siloxane ring and one in which the lithium atom is bound to an oxygen atom adjacent to an aluminium dopant atom, (Fig. 4a, b and Supplementary Figs. 21, 22) resulting in a disordered arrangement of lithium cations on the vermiculite surface. This has a strong impact on the ordering of the water contact layer, which we investigate by studying the probability density of finding water oxygen and hydrogen atoms at a given height above the vermiculite surface (Fig. $4 \mathrm{a}, \mathrm{b}$ ), as well as the orientational structuring of water in the 

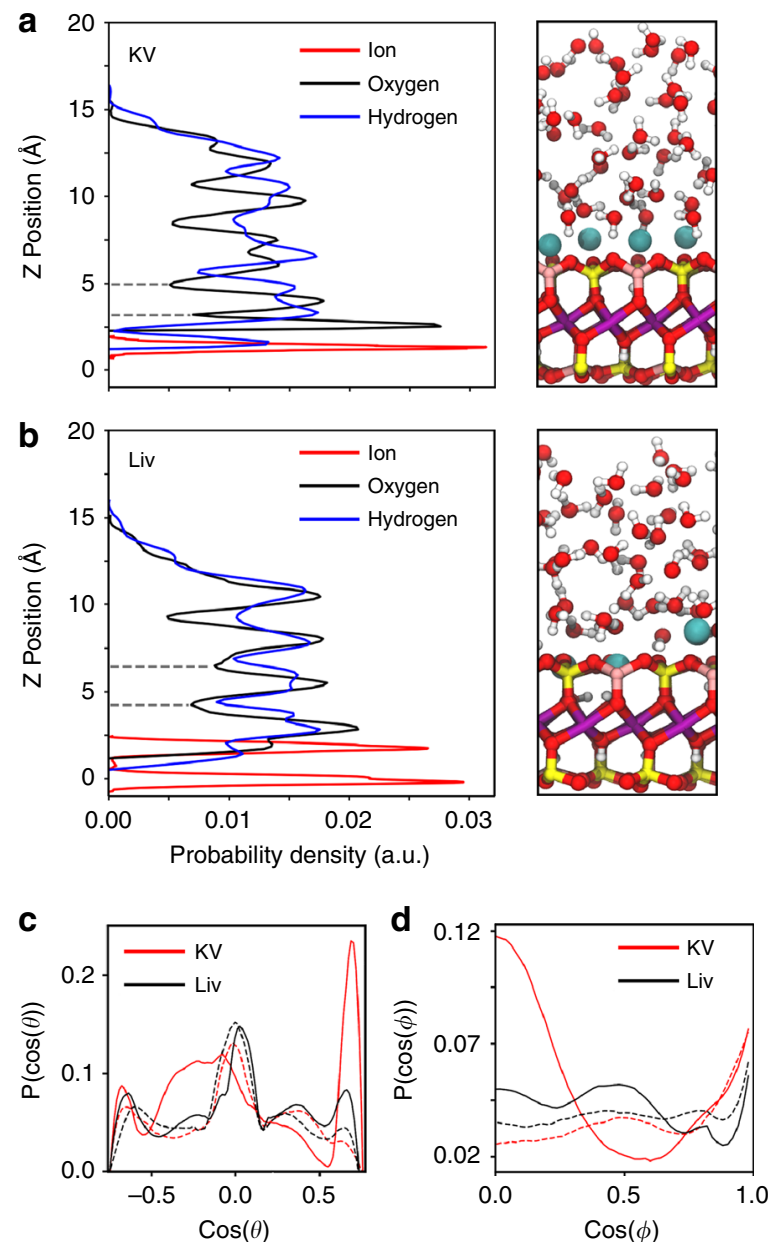

Fig. 4 Cation hydration structures on vermiculite surface. a, b Probability density plots for thin water films on vermiculite surfaces substituted with potassium and lithium, respectively. The $y$-axis zero is taken to be the mean $z$ position of the vermiculite surface oxygen atoms. Grey dotted lines indicate the distinction between the first and second contact layers of water. Snapshots are included showing the simulated liquid films and vermiculite structures on the right-hand side. Oxygen atoms are shown in red, hydrogen in white, silicon in yellow, aluminium in pink, magnesium in purple, and lithium and potassium in cyan. c Angular orientation of water molecules in the first and second contact layers of water using the angle between the vermiculite surface normal and water $\mathrm{O}-\mathrm{H}$ bonds $(\theta)$.

Distributions for the first contact layer are shown with solid lines, dashed lines represent the second layer of water. d Angular orientations of water molecules using the angle between the plane through the water molecule and the basal plane of the vermiculite surface $(\varphi)$. Distributions for the first contact layer are shown with solid lines, while the second contact layer is shown with dashed lines. All source data are provided as a Source Data file.

first and second contact layers (Fig. 4c, d). The contrast between the highly ordered $\mathrm{KV}$ contact layer, and the disordered LiV contact layer can be clearly seen in the plots of the probability density perpendicular to the surface (Fig. $4 \mathrm{a}, \mathrm{b}$ ), where the peaks in the first contact layer of water $(\sim 3 \AA$ above the vermiculite surface) in the probability density for water oxygen and hydrogen atoms are well defined and sharp in the case of KV but broad and poorly defined for $\mathrm{LiV}$ (below $\sim 4.5 \AA$ ). Most importantly, we note that unlike the contact layer, subsequent layers of water are far more ordered in the $\mathrm{LiV}$ case than in the case of $\mathrm{KV}$, a clear sign of a more hydrophilic surface. The effect of the structuring of the contact layer of water can be further investigated by studying the orientation of water molecules in the two contact layers
(Fig. 4c, d). We define two angles, $\theta$ as the angle between the vector normal to the vermiculite basal plane and the $\mathrm{O}-\mathrm{H}$ bond and $\varphi$ as the angle between the plane passing through the water molecule and the plane of the vermiculite surface (Supplementary Fig. 23). It can clearly be seen that the structure of the first contact layer in the KV system is strongly distorted from that of the second layer, with a preference for water molecules to orient with one $\mathrm{O}-\mathrm{H}$ bond directed downwards towards the vermiculite surface. This effect is due to the strong preference of $\mathrm{K}$ ions to bind above the vermiculite hydroxyl groups, thereby forcing water molecules to order around them. In the case of the LiV system, the orientations of water molecules in the first and second contact layers are extremely similar, indicating that the LiV surface allows the contact layer of water to orient in a similar way to the more 'bulk-like' layers above. This can be attributed to the large number of available water adsorption sites in the LiV case, as well as the alleviation of steric constraints due to some of the lithium ions adsorbing in the chelated configuration inside the vermiculite surface.

We propose that the KV surface is made more hydrophobic by the constraints induced by the structuring of water due to the small number of water adsorption sites available. This limited number of adsorption sites causes the interface between KV and water to be highly ordered and thus higher in energy. By comparison, the large number of available adsorption sites at the interface between LiV and water allows the interfacial water layer, and thus subsequent layers, to relax, making the surface more hydrophilic. We further show this by computing the binding energy of individual water molecules in the two contact layers for both systems. We find that in the KV case, the binding energy of water molecules in the contact layer is $-0.69 \pm 0.09 \mathrm{eV} / \mathrm{H}_{2} \mathrm{O}$, while in the lithium case water binds more strongly with an energy of $-0.89 \pm 0.02 \mathrm{eV} / \mathrm{H}_{2} \mathrm{O}$. This effect persists into the second contact layer, where the binding energies are found to be $-0.6 \pm 0.5 \mathrm{eV} / \mathrm{H}_{2} \mathrm{O}$ and $-0.7 \pm 0.5 \mathrm{eV} / \mathrm{H}_{2} \mathrm{O}$. The favourable binding of water molecules in the LiV contact layer explains the observed hydrophilicity and small contact angle of the $\mathrm{LiV}$ laminates.

In summary, we have revealed the cation controlled wetting properties of V-laminates, and in particular, we have successfully demonstrated the wetting transition of vermiculite laminates from superhydrophilic to hydrophobic by cation exchange. Our results show that in addition to ion charge and hydration energies, the atomic scale details of the hydration of ions can hugely affect the surface properties of solids and can be utilised for several applications. In addition, by making use of LiV's superhydrophilicity and stable hydration, we have demonstrated its unique opportunities in designing fouling-resistant oil separation membranes.

\section{Methods}

Fabrication of vermiculite laminates. Vermiculite dispersion was obtained from the thermally expanded vermiculite (Sigma Aldrich, UK) via a two-step ion exchange method as reported previously ${ }^{9,11} .50 \mathrm{mg}$ of vermiculite granules were added to $100 \mathrm{~mL}$ saturated $\mathrm{NaCl}(36 \mathrm{wt} \%)$ solution and stirred under refluxing at $100{ }^{\circ} \mathrm{C}$ for $24 \mathrm{~h}$ to replace the interlayer cations $\left(\mathrm{Mg}^{2+}\right)$ with $\mathrm{Na}^{+}$. The solution was then filtered out, and the collected vermiculite flakes were repeatedly washed with water and ethanol to remove any residual salt. Sodium exchanged bulk vermiculite was then dispersed in $100 \mathrm{~mL}$ of $2 \mathrm{M} \mathrm{LiCl}$ solution and refluxed for $12 \mathrm{~h}$ followed by filtration and extensive wash with water and ethanol. The filtered product was again dispersed in $100 \mathrm{~mL}$ fresh $2 \mathrm{M} \mathrm{LiCl}$ solution for another $12 \mathrm{~h}$ to secure a complete exchange. Lithium vermiculite (LiV) flakes so-obtained were sonicated in water for $20 \mathrm{~min}$ in order to exfoliate them into monolayer $\mathrm{LiV}$ flakes and subsequently centrifuged at $3000 \mathrm{rpm}$ to remove any multilayers and bulk residues left in the solution. The thickness and the lateral dimensions $(\sim 1 \times 1 \mu \mathrm{m})$ of the exfoliated vermiculite flakes (Supplementary Fig. 1) were measured by using Bruker Dimension Fast Scan AFM operating in peak force tapping mode. Electron microscopy characterisation of the flakes was carried out by using a FEI Talos 
F200X transmission electron microscope operating at an acceleration voltage of $200 \mathrm{kV}$.

The LiV-laminates of thickness $\approx 5 \mu \mathrm{m}$ were prepared by vacuum filtration of the LiV dispersion through a Whatman Anodisc alumina membrane filter $(0.2 \mu \mathrm{m}$ pore size and a diameter of 25 or $47 \mathrm{~mm}$ ). The resulting vermiculite films on alumina filters were peeled off from the substrate to obtain free-standing LiVlaminates (Fig. 1). The other cation exchanged V-laminates were prepared by immersing the LiV-laminate in $1 \mathrm{M}$ aqueous chloride solution of the desired cation for an hour.

GO laminates used for the comparison were also prepared by vacuum filtration as reported previously ${ }^{2}$. In brief, the aqueous suspension of GO prepared by bath sonication of graphite oxide flakes (purchased from BGT Materials Limited) were vacuum filtered through Anodisc alumina filters for obtaining free standing GO laminates. Cation-modified GO membranes for the contact angle measurements (Supplementary Fig. 4) were prepared as reported previously ${ }^{28}$ by immersing the GO membrane $(5 \mu \mathrm{m})$ inside $1 \mathrm{M}$ of the corresponding aqueous chloride solution for $1 \mathrm{~h}$ followed by rinsing with water.

Characterisation of vermiculite laminates. We have used inductively coupled plasma atomic emission spectrometry (ICP-AES), and X-ray diffraction (XRD) to characterise the V-laminates. The ICP-AES analysis provided the concentration of the interlayer cations in the $\mathrm{V}$-laminates and confirmed the efficient cation exchange. The samples for ICP-AES analysis were prepared by digesting the Vlaminate in a mixture of $1 \mathrm{ml}$ of $38 \%$ concentrated $\mathrm{HCl}$, and $1 \mathrm{ml}$ of $70 \%$ concentrated $\mathrm{HNO}_{3}$ for overnight. The samples were heated at $70{ }^{\circ} \mathrm{C}$ on a hot block in the tubes before being made up to $10 \mathrm{ml}$ with deionized water. The concentration of cations present in the $\mathrm{V}$-laminates was evaluated in moles per mg of the dry Vlaminate and is shown in Supplementary Table 1. Cation exchange method used here efficiently replaces the initial cations in the laminates with cations of interest. For example, the concentration of Li-ions in KV-laminate obtained by replacing $\mathrm{Li}$ ions with $\mathrm{K}$-ions is below the detection limit of the instrument (Supplementary Table 1). It should be noted that the $\mathrm{K}^{+}$-ion is present in all the laminates and the starting bulk vermiculite since its strong binding to the vermiculite surface ${ }^{26,33}$. Nevertheless, in KV-laminates the concentration of K-ions is significantly higher than that of other laminates.

XRD experiments were performed to study the laminar structure and swelling properties of the V-laminates with $5 \mu \mathrm{m}$ in thickness. We used Rigaku smart lab thin film XRD system ( $\mathrm{Cu}-\mathrm{Ka}$ radiation) operated at $1.8 \mathrm{~kW}$. For acquiring XRD from dry V-laminates, initially, all the samples were vacuum dried and stored in the glove box for $48 \mathrm{~h}$. The dehydrated samples were sealed in air-tight X-ray sample holder inside the glove box ${ }^{2,34}$ and taken out for further XRD measurements. Then the same laminates were exposed to ambient air $(\sim 40 \% \mathrm{RH})$ for $24 \mathrm{~h}$, and the measurements were repeated. For wet XRD, the laminates are soaked in the corresponding liquid for a minimum of $30 \mathrm{~min}$ and then immediately acquired the diffraction data.

Contact angle measurements. Sessile drop method (KRÜSS drop shape analyser, DSA100S) was used to measure the water contact angle on the laminates. Freestanding laminates were placed on a holey flat stage such a way that the central part of the laminate would be on top of the hole. A micro-syringe needle was used to precisely control the drop volume $(2 \mu \mathrm{l})$. The needle was lowered slowly until the drop touched the vermiculite laminates and then gently raised. The contact angle measurement module was operated in video mode at a capture speed of 60 frames per second.

Vermiculite-coated PA membranes. The vermiculite-coated PA membranes used for studying the antifouling oil-water/emulsion separation were prepared by filtering the vermiculite dispersion through a porous PA support $(170 \mu \mathrm{m}$ thick EMD Millipore $^{\mathrm{m}}$ PA hydrophilic membrane filters with $47 \mathrm{~mm}$ diameter) using a deadend pressure filtration system (Sterlitech HP4750, 1 bar of overpressure), followed by overnight vacuum drying. The vermiculite coating thicknesses of $15,30,45,60$, 75 , and $90 \mathrm{~nm}$ were fabricated. The coating thicknesses were estimated from the equation,

$$
t=\frac{C \times V}{A \times D}
$$

where $t$ is the thickness of the coating, $C$ is the concentration of the vermiculite dispersion, $V$ is the volume of the dispersion deposited, $A$ is the coating area, and $D$ is the density of vermiculite film. The density of the vermiculite film was obtained by measuring the weight and volume of a thick $(\approx 15 \mu \mathrm{m})$ freestanding V-laminate. To further confirm the thickness of the LiV coating on PA, we have conducted Hard X-ray Photoelectron Spectroscopy (HAXPES) on a $\sim 40 \mathrm{~nm}$ thick LiV-coated PA using a laboratory high-throughput HAXPES system from Scienta Omicron $\mathrm{GmbH}$, details of which are outlined in Ref. ${ }^{35}$. The system includes a gallium metal jet X-ray source (Excillum, Sweden), bespoke monochromator and EW-4000 electron energy analyser. The inelastic background modelling is performed via QUASES-Tougaard software ${ }^{36,37}$ (www.quases.com). Analysing the background of inelastically scattered electrons is a well-established technique for probing atomic distributions up to $\sim 20$ times as large as the inelastic electron mean free path (IMFP, $\lambda)^{38-40}$. One of the prime requirements to extend sampling depths over many tens of nm is a high energy X-Ray Source $(>5000 \mathrm{eV})$ as the IMFP of electron is governed by the kinetic energy of the electron ${ }^{41}$. Here using a $9.25 \mathrm{keV} \mathrm{Ga} \mathrm{Ka}$ metal jet X-ray source of the lab-based HAXPES, we captured the Si 1s peak and its background (or energy loss function) over hundreds of eV to higher binding energy (lower kinetic energy). Modelling of this background is then used to calculate the sample thickness. The two input parameters required for the inelastic background modelling are the inelastic mean free path and the inelastic scattering cross section, are calculated using the TPP-2M formula ${ }^{41}$.

Supplementary Fig. 24 shows the experimental Si-1s spectrum from the LiV deposited on a PA substrate and the modelled inelastic background using an inelastic mean path of $14.6 \mathrm{~nm}^{42}$. An Island model with $90 \%$ coverage of the vermiculite layer is assumed in the model. The fit to the experimental data reveals a silicate layer thickness of $37 \pm 3 \mathrm{~nm}$, consistent with the estimation based on the Eq. (1).

The bubble point pore size (largest pore size) of vermiculite-coated PA membranes was determined by using flow porometry technique (POROLUX $1000)^{43,44}$. The membranes were fully wetted with a low surface tension liquid (perfluoropolyether/porofil solution) and then sealed inside the sample chamber Nitrogen gas was allowed to flow into the chamber so as to force the wetting liquid out of the membrane pores. The pressure at which $\mathrm{N}_{2}$ gas overcomes the capillary pressure of the fluid and begins to flow through the wet sample yields the bubble point pore size ${ }^{43}$, which is given by,

$$
D=\frac{4 \gamma \cos \theta}{P} \approx \frac{4 \gamma}{P}
$$

where $D$ is the membrane pore size/diameter, and $\gamma$ is the surface tension of the wetting liquid, $P$ is the pressure, and $\theta$ is the contact angle of the liquid.

Hydrogel and polydopamine-coated PA membranes. Polydopamine was prepared by adding $0.2 \mathrm{wt} \%$ of dopamine hydrochloride in $10 \mathrm{mM}$ tris buffer solution at $\mathrm{PH} 8.5$ adjusted by $\mathrm{HCl}^{45}$. Hydrogel was prepared by a photoinitiated polymerisation process with acylamide, laponite, and 1-hydroxycyclohexyl phenyl ketone as the precursor, crosslinker, and initiator, respectively ${ }^{46}$. The chemicals were dissolved in water in 50:1.5:1 by weight, followed by UV ( $365 \mathrm{~nm})$ radiation for $2 \mathrm{~h}$. Both polydopamine and hydrogel coating are applied onto the PA membrane filter via immersing the membrane into the slurry for $4 \mathrm{~h}$.

Emulsion separation using LiV-coated membrane. The feed oil-in-water emulsion was prepared by dissolving $100 \mathrm{mg}$ of sodium dodecylbenzene sulfonate in $1 \mathrm{~L}$ of water followed by mixing with $1 \mathrm{~g}$ of kerosene/hexane/petroleum ether/vegetable oil. The mixture was sonicated and stirred for $1 \mathrm{~h}$ to obtain a stable (stable for a week) milky emulsion.

For the dead-end filtration, the emulsion was poured into the pressure vessel fitted with the membrane, and the permeate mass (at a feed pressure of 1 bar) was recorded every $30 \mathrm{~s}$ for a total of $30 \mathrm{~min}$ by a computer interfaced electronic scale (Ohaus, Navigator NV). After each $30 \mathrm{~min}$, the membrane was soaked in deionized water to remove the adsorbed oil droplets from the surface of the membrane and repeated the emulsion separation. This process is carried out for 15 cycles, and the permeate flux is continuously monitored as shown in Supplementary Fig. 9.

The cross-flow emulsion separation was carried out using the Armfield FT17 crossflow filtration system. Before filtering the emulsion, deionized water was filtered through the membrane $\left(1 \mathrm{~cm}^{2}\right.$ area) for $6 \mathrm{~h}$ until a steady water flux was obtained. The emulsion was then poured into the feed tank and filtered through the membrane under a feed pressure of 1 bar and a cross-flow speed of $0.05 \mathrm{~m} \mathrm{~s}^{-1}$ to $0.5 \mathrm{~m} \mathrm{~s}^{-1}$ (Supplementary Fig. 10). This process was carried out for $15 \mathrm{~h}$ continuously and the permeate mass was recorded by a computer interfaced electronic scale (Ohaus, Navigator NV).

The filtration performance of the membranes for both dead-end and cross flow filtration was characterised in terms of the permeate flux (Fig. $3 \mathrm{c}$ and Supplementary Fig. 9) and total organic content in the permeate. During the deadend filtration, LiV-coated PA and the bare PA membrane have an initial permeate flux of $\approx 6500 \mathrm{~L} \mathrm{~m}^{-2} \mathrm{~h}^{-1}$, and it decreases by $90 \%$ in the first $30 \mathrm{~min}$ of the filtration process. However, the initial permeate flux got fully recovered for the LiV-coated PA membrane in the consecutive cycles of filtration after a simple water rinsing (Supplementary Fig. 9). The flux reduction and its recovery in each cycle of filtration is due to the deposition of the oil droplets on the surface of the membrane and its removal by water soaking, respectively. On the other hand, the initial permeate flux continuously declined for the bare PA after each cycle of filtration and reduced to $\approx 10 \%$ of the initial permeate flux after the 15 th cycle (Fig. $3 \mathrm{c}$ ).

The amount of oil permeated through the membrane is measured by using the total organic carbon (TOC) content analyser (Shimadzu TOC-VCPN analyser). The separation efficiency of the LiV-coated membrane was calculated by oil rejection $(R)$ given by

$$
R=\left(1-\frac{C_{p}}{C_{f}}\right) \times 100
$$

where $C_{p}$ and $C_{f}$ are the total carbon content of the collected permeate and the feed emulsion, respectively. Supplementary Fig. 14 shows the oil rejection for the LiVcoated PA membrane for different type emulsions prepared from different oils. 
Salty water emulsion filtration test was carried out using crossflow filtration with a crossflow speed of $0.05 \mathrm{~m} \mathrm{~s}^{-1}$ at 1 bar pressure. Before adding the salt, both the coated and the non-coated membranes underwent $15 \mathrm{~h}$ of continuous emulsion filtration so that the flux reached a steady-state. $\mathrm{NaCl}$ or $\mathrm{KCl}$ salt was then slowly added into the feed tank until the required salt solution concentration is reached (Supplementary Fig. 15). Thereafter the permeate flux during the crossflow filtration was recorded continuously for $100 \mathrm{~min}$.

Wetting properties of LiV-coated PA membranes. KRÜSS drop shape analyser (DSA100S) was used to carry out all the wetting measurements. The wetting time of the LiV-coated PA membrane was studied by recording the time taken to fully spread $2 \mu \mathrm{L}$ of water dropped on the surface of the membrane ${ }^{30}$. For the precise measurement of wetting time, a high-resolution video camera was operated at a capture speed of 60 frames per second. For example, the wetting time for a $2 \mu \mathrm{L}$ water droplet added to the LiV-coated PA membrane with a pore size of $\sim 1 \mu \mathrm{m}$ (coating thickness of $\sim 30 \mathrm{~nm}$ ) was found to be $\sim 0.8 \mathrm{~s}$ whereas the droplet on bare PA took $\sim 1.4 \mathrm{~s}$ to get it spread completely (Supplementary Fig. 8a). Supplementary Fig. $8 \mathrm{~b}$ shows the variation in wetting time as a function of LiV coating thickness or the pore size of the LiV-coated membrane. It was found that $\sim 30 \mathrm{~nm}$ is the optimum LiV coating thickness required for the shortest wetting time. The higher coating thickness leads to smaller pore size and lower surface roughness and hence longer wetting time. For coating with thickness $\leq 30 \mathrm{~nm}$, we did not find any significant changes in root mean square roughness of the membrane with respect to bare PA membrane (Supplementary Fig. 25). This is mainly due to the flexible nature of the $2 \mathrm{D}$ flakes of vermiculites, which helps to follow the texture of the underlying PA membrane.

The underwater contact angle measurements were performed as reported previously ${ }^{47}$. A custom-made quartz cell was employed as the water reservoir, where the LiV-coated membrane was fixed with coated-side-down on top of the container. The bottom of the cell was connected to a micro-syringe, where the oil droplets were released and floated to the surface of the membrane. The underwater oil contact angle of bare PA was found to be $150 \pm 4^{\circ}$, whereas it increases to $168 \pm$ $3^{\circ}$ after coating with $30 \mathrm{~nm} \mathrm{LiV} \mathrm{(Supplementary} \mathrm{Fig.} \mathrm{18).}$

Same setup for the underwater contact angle was employed for measuring the roll-off angle. For the roll-off angle test, an oil droplet $(10 \mu \mathrm{l})$ was squeezed out from the micro-syringe to the surface of the sample immersed in water. The sample stage was then slowly tilted $\left(10^{\circ} \mathrm{min}^{-1}\right)$ until the oil droplet slide off from its original position. The whole process was recorded every $400 \mathrm{~ms}$ with the aid of a high-resolution camera, and the roll-off angle is obtained by monitoring the movement of the droplet with respect to its original position (Supplementary Figs. 11, 16).

Oil adhesion force measurement. Under-water oil adhesion forces were measured as reported previously ${ }^{48}$ using a high sensitivity microelectromechanical balance system (DCAT250, Data Physics). The whole measurement process involves three major steps, namely advancing, contacting and receding. The forces were recorded during the entire step using a high sensitivity microelectromechanical balance. First, an oil droplet $(10 \mu \mathrm{L})$ was suspended on a metal cap attached to the balance. During the advancing process (step 1 in Fig. 3d), the membrane samples immersed in the water attached to a stage is lifted up slowly at a speed of $0.05 \mathrm{~mm} \mathrm{~s}^{-1}$ until the membrane surface contacts the oil droplet. After contacting, a fixed pre-load $(10 \mu \mathrm{N})$ was applied (step 2 in Fig. 3d). The stage then moves downwards with a speed of $0.02 \mathrm{~mm} \mathrm{~s}^{-1}$ (receding, step 3 in Fig. 3d), leading to the detachment of the oil droplet from the sample surface. During this detachment process, at a certain position, the droplet completely detaches from the sample surface indicated by a sudden drop in the force curve (step 4 in Fig. 3d). The change in the force during this sudden detachment step was taken as the maximum adhesion force. For bare PA samples, this force is found to be $35.4 \pm 3.5$ $\mu \mathrm{N}$. However, for LiV-coated PA, no apparent sudden drop in the force was noticed suggesting a negligible adhesion force ${ }^{48}$ (below the detection limit of $1 \mu \mathrm{N}$ ). We used 1,2-dichloroethane, hexane, and toluene to replicate the oil for these measurements and did not notice any significant differences between different solvents.

Influence of ionic strength on contact angle. To study the impact of surface charge density on the contact angle of V-laminates, we measured the contact angle at different concentrations $(0.0001-1 \mathrm{M})$ of salt solutions. It is known that at high ionic strength, the surface charge density decreases due to the compression of the electric double layer ${ }^{49}$ and hence studying the contact angle variation with ionic strength directly probe its dependence on surface charge density. These measurements were carried out by investigating the contact angle of different V-laminates using a salt solution of varying concentrations. To avoid any ion exchange during the contact angle measurements, we used the salt solution corresponds to the interlayer cations of the $\mathrm{V}$-laminates (e.g., $\mathrm{LiCl}$ solution for $\mathrm{LiV}$-laminate and $\mathrm{KCl}$ for KV-Laminates). Supplementary Fig. 19 shows the contact angle as a function of the concentration of salt solution for five different V-laminates. For all five types of $\mathrm{V}$-laminates, contact angle did not show any significant change with the ionic strength. It should also be noted that, for $\mathrm{LaV}$ and $\mathrm{SnV}$ laminates, the contact angle slightly changes at higher concentration of the salt solution. This is due to the change in $\mathrm{pH}$ (salt solution become more acidic) of the salt solution at these concentrations.

Zeta potential measurements. To further confirm the absence of correlation between surface charge density and the contact angle, we have measured the zeta potential of different $\mathrm{V}$-laminates and its ionic strength dependence via the streaming potential technique (Anton Paar SurPASS3). These measurements were carried out by placing two V-laminates inside the measuring cell forming a capillary with $100 \mu \mathrm{m}$ height. Then the test liquid (a mixture of $\mathrm{LiCl}$ and $\mathrm{KCl}$ ) with known ionic strength was injected through the capillary at a specific pressure (200-600 mbar), and the potential difference was measured between the two ends of the streaming channel as the streaming potential. We used mixed solutions of $0.01 \mathrm{M} \mathrm{LiCl}$ and $\mathrm{KCl}$ as the standard electrolytes for streaming potential measurements since 1:1 LiCl:KCl electrolyte is considered inert with respect to surface interaction ${ }^{50}$. In addition, since the time for each measurement is very short $(<30$ s), the effect of ion exchange is minimal.

For samples with a planar surface, its zeta potential can be related with the streaming potential by Helmholtz-Smoluchowski equation ${ }^{50,51}$.

$$
\xi=\frac{d U_{s t r}}{d \Delta p} \times \frac{\eta}{\varepsilon \times \varepsilon_{\mathrm{o}}} \times \kappa
$$

where $U_{s t r}$ is the measured streaming potential at a specific cross-capillary pressure $\Delta p, \kappa$ is the conductivity of the capillary, and $\eta$ and $\varepsilon \times \varepsilon_{\mathrm{o}}$ are the viscosity and dielectric coefficient of the electrolyte solution.

Supplementary Fig. 19b shows the zeta potential for different V-laminates. We found that LiV has the maximum zeta potential and it decreases to nearly zero for $\mathrm{LaV}$ and $\mathrm{SnV}$ with multivalent ions as the interlayer cations. This could be due to the charge neutralisation of the negatively charged silicate layer with positively charged cations ${ }^{52}$. Even though the variation of zeta potential qualitatively correlates with the change in contact angle (Laminates with higher zeta potential shows minimum contact angle), the large change in the contact angle for LiVlaminates compared with other laminates was not correlating with the zeta potential measurements. This absence of correlation between the zeta potential and the contact angle is further confirmed by measuring the ionic strength dependence of zeta potential. As shown in Supplementary Fig. 19b, the zeta potential decreases with ionic strength as expected, whereas the contact angle is largely independent of ionic strength.

Surface free energy estimation. Fowkes' surface energy theory ${ }^{53,54}$ describes the contact angle $(\theta)$ of a liquid on a solid surface as

$$
\frac{\gamma_{l}(\cos \theta+1)}{2}=\left(\gamma_{l}^{d}\right)^{1 / 2}\left(\gamma_{s}^{d}\right)^{1 / 2}+\left(\gamma_{l}^{p}\right)^{1 / 2}\left(\gamma_{s}^{p}\right)^{1 / 2}
$$

where $\gamma_{l}$ is surface tension of the liquid, $\gamma_{l}^{d}, \gamma_{l}^{p}$ are the dispersive and polar component of the surface tension of the wetting liquid, respectively, and $\gamma_{s}^{d}, \gamma_{s}^{p}$ are the dispersive and polar component of the solid surface energy, respectively. The surface energy component of the laminates can be determined from Eq. (5) by using the contact angle measurements with liquids of known polar and dispersive components ${ }^{55}$. We used the contact angle data of a nonpolar aprotic liquid (diiodomethane ${ }^{56}, \gamma_{1}=50.8 \mathrm{~m} \mathrm{Nm}^{-1}$, $\gamma_{1} \mathrm{p}=0$, and $\gamma_{1}^{\mathrm{d}}=50.8 \mathrm{~m} \mathrm{Nm}^{-1}$ ) and a polar protic liquid (water ${ }^{56}, \gamma_{1}=72.8 \mathrm{~m} \mathrm{Nm}^{-1}$, $\gamma_{1} \mathrm{p}=51 \mathrm{~m} \mathrm{Nm}^{-1}$, and $\gamma_{1}^{\mathrm{d}}=21.8 \mathrm{~m} \mathrm{Nm}^{-1}$ ) on the V-laminates (Supplementary Table S2) and calculated the $\gamma_{s}^{d}$ and $\gamma_{s}^{p}$ (Supplementary Fig. 20). The total surface energy $\left(\gamma_{s}\right)$ of the V-laminate can be calculated as in Eq. (6).

$$
\gamma_{s}=\gamma_{s}^{d}+\gamma_{s}^{p}
$$

Density functional theory (DFT) geometry optimisations. Spin polarised DFT calculations were performed using the $\mathrm{CP} 2 \mathrm{~K}^{57}$ software package using the Quickstep algorithm, with the PBE-D3 ${ }^{58,59}$ functional, Goedecker-Teter-Hutter pseudopotentials ${ }^{60,61}$, a 350 Ry plane-wave cut-off, a relative cut-off of 60 Ry and a DZVP molecularly optimised basis set $^{62}$.

The crystal structure of the bare vermiculite surface was optimised by first optimising the unit cell volume of the vermiculite structure, until the total energy is converged to within $10^{-4} \mathrm{eV}$, this was followed by an optimisation of the ionic positions until the total energy was converged to $10^{-4} \mathrm{eV}$. We study a model system in which $25 \%$ of the silicon atoms in the basal plane of the Vermiculite structure have been substituted with aluminium, resulting in a net surface charge of -2 e per unit cell, which is representative of that found in natural vermiculite ${ }^{33}$. In each case, we place a charge equalising number of cations on the vermiculite surface.

Liquid water film AIMD simulations. Water films on vermiculite surfaces were studied for $\mathrm{Li}$ and $\mathrm{K}$ using the same structural model as discussed above. Four independent vermiculite surfaces were prepared for each ion in combinations of their vacuum optimised locations. A $2 \times 1$ supercell of vermiculite was used for AIMD simulations, with lattice parameters in the in-plane direction of $10.62 \times 9.20$ $\AA$. $30 \AA$ of vacuum was introduced to these above the bare vermiculite surface. The surface was then hydrated with 40 molecules of $\mathrm{D}_{2} \mathrm{O}$. Deuterated water is used to lessen the need to consider nuclear quantum effects and to allow for the use of a 
time step of $0.5 \mathrm{fs}$. Replica simulations were performed in the NVT ensemble at $300 \mathrm{~K}$, using a coloured noise thermostat ${ }^{63}$ and were equilibrated for 5 ps before statistics were collected for analysis. After optimisation and equilibration, there remained $\sim 20 \AA$ of vacuum between the surface of the liquid film and the periodic image of the vermiculite surface. In total, $35 \mathrm{ps}$ of simulation time was analysed for the $\mathrm{K}$ system, and $40 \mathrm{ps}$ for the lithium system.

Angular distributions of water molecules. We employ two metrics to study the angular distribution of water molecules above the vermiculite surface. We denote the angle between the direction of the $\mathrm{O}-\mathrm{H}$ bonds of the water molecules and the surface normal to the vermiculite surface as $\theta$. We also compute the angle between the vermiculite basal plane and the plane passing through all three atoms of the water molecule, which we denote as $\varphi$. This is illustrated in Supplementary Fig. 23.

Water binding energy calculations. Water binding energies were estimated for water molecules in the first and second contact layers of water. This was done by performing independent DFT geometry optimisations on snapshots selected from the AIMD simulations. Ten snapshots for the first and second layer for both $\mathrm{Li}$ and $\mathrm{K}$ ions were selected. The binding energy is then defined as

$$
E_{b}=E_{\text {tot }}-\left(N_{\text {wat }} * E_{\text {wat }}+E_{\text {surf }}\right),
$$

where $E_{t o t}$ is the total energy of the optimised surface with either one or two contact layers of water, $N_{\text {wat }}$ is the number of water molecules in the contact layers, $E_{\text {wat }}$ is the total energy of the isolated water molecule in vacuum and $E_{\text {surf }}$ is the total energy of the optimised vermiculite surface with ions.

\section{Data availability}

The data underlying Figs. 1b, 2b, 3b-d, 4a-d, Supplementary Figures 2, 3, 6, 8b, 9, 10, 12, $13,14,15,16,17,19,20,22$, and 24 are provided as source file. All other data are available from the corresponding author upon reasonable request.

Received: 23 August 2019; Accepted: 7 February 2020;

Published online: 27 February 2020

\section{References}

1. Espinosa-Marzal, R. M. Interactions in water across interfaces: from nano to macro-scale perspective. In: Mercury L., Tas N., Zilberbrand M. (eds) Transport and Reactivity of Solutions in Confined Hydrosystems. (Springer, Dordrecht, 2014).

2. Abraham, J. et al. Tunable sieving of ions using graphene oxide membranes. Nat. Nanotechnol. 12, 546-550 (2017).

3. Siria, A., Bocquet, M.-L. \& Bocquet, L. New avenues for the large-scale harvesting of blue energy. Nat. Rev. Chem. 1, 0091 (2017).

4. Jungwirth, P. \& Winter, B. Ions at aqueous interfaces: From water surface to hydrated proteins. Annu. Rev. Phys. Chem. 59, 343-366 (2008).

5. Grim, R. E. Clay mineralogy. Science 135, 890-898 (1962).

6. Karaborni, S., Smit, B., Heidug, W., Urai, J. \& van Oort, E. The swelling of clays: molecular simulations of the hydration of montmorillonite. Science 271, 1102-1104 (1996).

7. Aguzzi, C., Cerezo, P., Viseras, C. \& Caramella, C. Use of clays as drug delivery systems: possibilities and limitations. Appl. Clay Sci. 36, 22-36 (2007).

8. Ismadji, S., Soetaredjo, F. E. \& Ayucitra, A. Clay Materials for Environmental Remediation. (Springer International Publishing, Switzerland, 2015).

9. Walker, G. F. \& Garrett, W. G. Chemical exfoliation of vermiculite and the production of colloidal dispersions. Science 156, 385-387 (1967).

10. Li, J. \& Whittingham, M. S. Preparation and characterization of lithium, manganese, cobalt and copper vermiculites. Mater. Res. Bull. 26, 849-856 (1991).

11. Shao, J. J., Raidongia, K., Koltonow, A. R. \& Huang, J. Self-assembled twodimensional nanofluidic proton channels with high thermal stability. Nat. Commun. 6, 7602 (2015)

12. Schaetzl, R. J. \& Thompson, M. L. Basic concepts: soil mineralogy. Soils: Genesis and Geomorphology. Cambridge University Press, 2015.

13. Shang, J., Flury, M., Harsh, J. B. \& Zollars, R. L. Contact angles of aluminosilicate clays as affected by relative humidity and exchangeable cations. Colloid Surf. A 353, 1-9 (2010).

14. Ballah, J. et al. Effect of chemical and geometrical parameters influencing the wettability of smectite clay films. Colloid Surf. A 511, 255-263 (2016).

15. Yin, X., Gupta, V., Du, H., Wang, X. \& Miller, J. D. Surface charge and wetting characteristics of layered silicate minerals. Adv. Colloid Interface Sci. 179-182, 43-50 (2012).
16. JańczukEmil, B., Chibowski, E., Hajnos, M., Białopiotrowicz, T. \& Stawiński, J. Influence of exchangeable cations on the surface free energy of kaolinite as determined from contact angles. Clay Clay Min. 37, 269-272 (1989).

17. Verdaguer, A., Sacha, G. M., Bluhm, H. \& Salmeron, M. Molecular structure of water at interfaces: wetting at the nanometer scale. Chem. Rev. 106, 1478-1510 (2006).

18. Koopal, L. K. Wetting of solid surfaces: fundamentals and charge effects. Adv. Colloid Interface Sci. 179-182, 29-42 (2012).

19. Mugele, F. et al. Ion adsorption-induced wetting transition in oil-water-mineral systems. Sci. Rep. 5, 10519 (2015).

20. Chu, Z., Feng, Y. \& Seeger, S. Oil/water separation with selective superantiwetting/superwetting surface materials. Angew. Chem. Int. Ed. 54, 2328-2338 (2015).

21. Gupta, R. K., Dunderdale, G. J., England, M. W. \& Hozumi, A. Oil/water separation techniques: a review of recent progresses and future directions. $J$. Mater. Chem. A 5, 16025-16058 (2017).

22. Zhu, Y., Wang, D., Jiang, L. \& Jin, J. Recent progress in developing advanced membranes for emulsified oil/water separation. NPG Asia Mater. 6, e101 (2014).

23. Liu, M., Wang, S. \& Jiang, L. Nature-inspired superwettability systems. Nat. Rev. Mater. 2, 17036 (2017)

24. Rana, D. \& Matsuura, T. Surface modifications for antifouling membranes. Chem. Rev. 110, 2448-2471 (2010).

25. Norrish, K. The swelling of montmorillonite. Discuss. Faraday Soc. 18, 120-134 (1954).

26. Minker-Villemin, C., Bowen, P., Lemaître, J. \& Ring, T. A. Preparation and properties of potassium-vermiculite films. J. Mater. Res. 13, 228-236 (1998).

27. Jones, D. J., Roziere, J., Olivera-Pastor, P., Rodriguez-Castellon, E. \& JimenezLopez, A. Local environment of intercalated lanthanide ions in vermiculite. J. Chem. Soc. Faraday Trans. 87, 3077-3081 (1991).

28. Chen, L. et al. Ion sieving in graphene oxide membranes via cationic control of interlayer spacing. Nature 550, 380 (2017).

29. He, K. et al. Cleaning of oil fouling with water enabled by zwitterionic polyelectrolyte coatings: overcoming the imperative challenge of oil-water separation membranes. ACS Nano 9, 9188-9198 (2015).

30. Kota, A. K., Kwon, G., Choi, W., Mabry, J. M. \& Tuteja, A. Hygroresponsive membranes for effective oil-water separation. Nat. Commun. 3, 1025 (2012)

31. Yi, H. et al. Ultrafiltration membranes with structure optimized graphene oxide coatings for antifouling oil/water separation. Adv. Mater. Interfaces 2, 1400433 (2015).

32. Good, R. J. Surface free energy of solids and liquids: thermodynamics, molecular forces, and structure. J. Colloid Interface Sci. 59, 398-419 (1977).

33. Sposito, G. et al. Surface geochemistry of the clay minerals. Proc. Natl Acad. Sci. USA 96, 3358-3364 (1999).

34. Yang, Q. et al. Ultrathin graphene-based membrane with precise molecular sieving and ultrafast solvent permeation. Nat. Mater. 16, 1198 (2017).

35. Regoutz, A. et al. A novel laboratory-based hard X-ray photoelectron spectroscopy system. Rev. Sci. Instrum. 89, 073105 (2018).

36. Tougaard, S. Surface nanostructure determination by X-ray photoemission spectroscopy peak shape analysis. J. Vac. Sci. Technol. A 14, 1415 (1996).

37. Tougaard, S. Inelastic background correction and quantitative surface analysis. J. Electron Spectros. Relat. Phenom. 52, 243-271 (1990).

38. Tougaard, S. Accuracy of the non-destructive surface nanostructure quantification technique based on analysis of the XPS or AES peak shape. Surf. Interface Anal. 26, 249-269 (1998).

39. Cui, Y.-T. et al. Thickness and structure of thin films determined by background analysis in hard X-ray photoelectron spectroscopy. J. Appl. Phys. 121, 225307 (2017)

40. Tougaard, S. Energy loss in XPS: fundamental processes and applications for quantification, non-destructive depth profiling and 3D imaging. J. Electron Spectros. Relat. Phenom. 178-179, 128-153 (2010).

41. Tanuma, S., Powell, C. J. \& Penn, D. R. Calculations of electron inelastic mean free paths. IX. Data 41 elemental solids $50 \mathrm{eV} 30 \mathrm{keV}$ range. Surf. Interface Anal. 43, 689-713 (2011).

42. Shinotsuka, H., Tanuma, S., Powell, C. J. \& Penn, D. R. Erratum: Calculations of electron inelastic mean free paths. X. Data for 41 elemental solids over the $50 \mathrm{eV}$ to $200 \mathrm{keV}$ range with the relativistic full Penn algorithm. Surf. Interface Anal. 47, 1132 (2015).

43. Jena, A. \& Gupta, K. Pore volume of nanofiber nonwovens. Int. Nonwovens J. 14, 25-30 (2005).

44. Yu, W. K., Chung, T.-S. \& Gryta, M. Hydrophobic PVDF hollow fiber membranes with narrow pore size distribution and ultra-thin skin for the fresh water production through membrane distillation. Chem. Eng. Sci. 63 , 2587-2594 (2008).

45. Lee, H., Dellatore, S. M., Miller, W. M. \& Messersmith, P. B. Mussel-inspired surface chemistry for multifunctionalcoatings. Science 318, 426-430 (2007). 
46. Haraguchi, K. \& Takada, T. Synthesis and characteristics of nanocomposite gels prepared by in situ photopolymerization in an aqueous system. Macromolecules 43, 4294-4299 (2010). 9.

47. Hejazi, V., Nyong, A. E., Rohatgi, P. K. \& Nosonovsky, M. Wetting transitions in underwater oleophobic surface of brass. Adv. Mater. 24, 5963-5966 (2012).

48. Cheng, Q. et al. An underwater pH-responsive superoleophobic surface with reversibly switchable oil-adhesion. Soft Matter 8, 6740-6743 (2012).

49. Brown, M. A., Goel, A. \& Abbas, Z. Effect of electrolyte concentration on the stern layer thickness at a charged interface. Angew. Chem. Int. Ed. 55, 3790-3794 (2016).

50. Luxbacher, T. The Zeta Potential for Solid Surface Analysis. (Anton Paar $\mathrm{GmbH}$, Austria, 2014).

51. Werner, C., Körber, H., Zimmermann, R., Dukhin, S. \& Jacobasch, H.-J. Extended electrokinetic characterization of flat solid surfaces. J. Colloid Interface Sci. 208, 329-346 (1998).

52. Velde, B. B. \& Meunier, A. The Origin of Clay Minerals in Soils and Weathered Rocks. (Springer-Verlag Berlin, Heidelberg, 2008).

53. Fowkes, F. M. Attractive forces at interfaces. Ind. Eng. Chem. 56, 40-52 (1964).

54. Fowkes, F. M. Additivity of intermolecular forces at interfaces. I. determination of the contribution to surface and interfacial tensions of dispersion forces in various liquids. J. Phys. Chem. 67, 2538-2541 (1963).

55. Kozbial, A. et al. Study on the surface energy of graphene by contact angle measurements. Langmuir 30, 8598-8606 (2014).

56. Cwikel, D., Zhao, Q., Liu, C., Su, X. \& Marmur, A. Comparing contact angle measurements and surface tension assessments of solid surfaces. Langmuir 26, 15289-15294 (2010).

57. Hutter, J., Iannuzzi, M., Schiffmann, F. \& VandeVondele, J. cp2k: atomistic simulations of condensed matter systems. Wiley Interdiscip. Rev. Comput. Mol. 4, 15-25 (2013).

58. Grimme, S., Antony, J., Ehrlich, S. \& Krieg, H. A. consistent and accurate ab initio parametrization of density functional dispersion correction (DFT-D) for the 94 elements H-Pu. J. Chem. Phys. 132, 154104 (2010).

59. Grimme, S. Semiempirical GGA-type density functional constructed with a long-range dispersion correction. J. Comput. Chem. 27, 1787-1799 (2006).

60. Krack, M. Pseudopotentials for $\mathrm{H}$ to $\mathrm{Kr}$ optimized for gradient-corrected exchange-correlation functionals. Theor. Chem. Acc. 114, 145-152 (2005)

61. Goedecker, S., Teter, M. \& Hutter, J. Separable dual-space Gaussian pseudopotentials. Phys. Rev. B 54, 1703-1710 (1996).

62. VandeVondele, J. \& Hutter, J. Gaussian basis sets for accurate calculations on molecular systems in gas and condensed phases. J. Chem. Phys. 127, 114105 (2007).

63. Ceriotti, M., Bussi, G. \& Parrinello, M. Colored-noise thermostats à la carte. $J$. Chem. Theory Comput. 6, 1170-1180 (2010).

\section{Acknowledgements}

This work was supported by the Royal Society, Engineering and Physical Sciences Research Council, UK (EP/K016946/1 and EP/N013670/1), British Council (award reference number 279336045), Graphene Flagship, and European Research Council (contract 679689). We thank Mr. Paul R. Lythgoe at Manchester Analytical Geochemistry Unit, School of Earth and Environmental Sciences, the University of Manchester for assisting with ICP measurements. HAXPES and XRD were performed in the Henry Royce Institute for Advanced Materials, funded through EPSRC grants EP/R00661X/1, EP/P025021/1 and EP/P025498/1. We thank Ben Spencer for helping with HAXPES measurements. A.M. acknowledges support from the European Research Council under the European Union's Seventh Framework Programme (FP/2007-2013)/ERC Grant Agreement No. 616121 (HeteroIce project). We are grateful for computational support from the UK Materials and Molecular Modelling Hub, which is partially funded by EPSRC (EP/P020194/1), for which access was obtained via the UKCP consortium and funded by EPSRC grant ref EP/P022561/1. The authors acknowledge the use of the facilities at UCL Grace High Performance Computing Facility (Grace@UCL), and associated support services.

\section{Author contributions}

R.R.N., K.H., C.T.C. designed the project, and R.R.N. directed it. K.H. performed the experiments and analysed the data with help from C.T.C. and R.R.N. P.R. and A.M. performed the theoretical modelling and simulations. T.B. and C.C. performed cross-flow filtration experiments. V.S., K.-G.Z., and Y.S. helped in sample preparation, characterisation and data analysis. E.P. carried out the electron microscopy characterisation. P.B.P. performed XPS analysis. R.R.N., K.H., C.T.C., P.R., and A.M. co-wrote the paper. All authors contributed to discussions.

\section{Competing interests}

The authors declare no competing interests.

\section{Additional information}

Supplementary information is available for this paper at https://doi.org/10.1038/s41467020-14854-4.

Correspondence and requests for materials should be addressed to C.T.C., A.M. or R.R.N

Peer review information Nature Communications thanks Chuanhua Duan, Marco Ortenzi, and other anonymous reviewers for their contributions.

Reprints and permission information is available at http://www.nature.com/reprints

Publisher's note Springer Nature remains neutral with regard to jurisdictional claims in published maps and institutional affiliations.

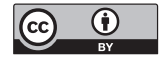

Open Access This article is licensed under a Creative Commons Attribution 4.0 International License, which permits use, sharing, adaptation, distribution and reproduction in any medium or format, as long as you give appropriate credit to the original author(s) and the source, provide a link to the Creative Commons license, and indicate if changes were made. The images or other third party material in this article are included in the article's Creative Commons license, unless indicated otherwise in a credit line to the material. If material is not included in the article's Creative Commons license and your intended use is not permitted by statutory regulation or exceeds the permitted use, you will need to obtain permission directly from the copyright holder. To view a copy of this license, visit http://creativecommons.org/ licenses/by/4.0/.

(c) The Author(s) 2020 